\title{
Design and Implementation of the 1024-QAM RF Transmission System for UHD Cable TV Broadcasting
}

\author{
Sung-Hoon Kim ${ }^{1}$, Jinsoo Choi ${ }^{1}$, Jinwoong Kim ${ }^{1}$, Md. Sazzad Hossen ${ }^{2}$, and Ki-Doo Kim ${ }^{2}$ \\ Electronics and Telecommunications Research Institute, KOREA ${ }^{1}$ \\ Kookmin University, KOREA ${ }^{2}$ \\ *contact : steve-kim@etri.re.kr
}

\begin{abstract}
This paper presents the 1024-QAM transmission system design, implementation and performance for providing UHDTV services over HFC network. The CNR@TOV (Threshold Of Visibility, BER of $3.6 \mathrm{e}-6$ ) of proposed system is $32.7 \mathrm{~dB}$ under AWGN and data throughput is $48.54 \mathrm{Mbps}$ within $6 \mathrm{MHz}$ bandwidth. In this paper, we provide a detail description of UHDTV(Ultra High Definition TV) 1024-QAM cable transmission system design which includes functional description of modem components, simulation results, and lab test results for performance evaluation. To overcome severe burst noise and multipath channel impairments, we proposed the MPEG transport stream layer FEC (TS-FEC) and pilot symbol insertion for additional error control coding gain, fast channel acquisition and estimation.
\end{abstract}

Keywords - UHDTV, J.83 Annex B, 1024-QAM, Transport Stream Layer FEC(TS-FEC)

\section{INTRODUCTION}

The OpenCable(J.83 Annex B) standard adopts single carrier QAM modulation schemes from 64-QAM to 256QAM for CATV broadcasting services, as well as for data transmissions[1]. Responding to increased consumer demand for broader range of digital TV services, many cable operators already upgraded their networks several years ago, and increased the frequency range used for downstream transmission up to its maximum of $1 \mathrm{GHz}$. As a result, most cable operators currently offer a rich analog TV package and a hundred digital TV channels and increasing amount of new interactive, high quality video type services. When analog channels will be digitalized, new channels will be introduced and cable operators will start offering realistic broadcasting and bandwidth demanding services such as UHDTV in order to boost digital TV penetration in their networks. At the same time, the introduction of $4 \mathrm{~K}-\mathrm{UHD}(3840 \times 2160)$, $8 \mathrm{~K}-$ UHD $(7680 \times 4320)$ band intensive realistic broadcasting TV services will generate significant capacity problems in the coaxial parts of hybrid fiber coax networks[2].

For this reason, 1024-QAM modulation schemes for UHDTV have been proposed in previous researches[3][4]. However, two points have not been addressed in previous researches. One is a burst noise handling issue and the other is a practical implementation issue.
To resolve the issues not addressed in previous researches, we propose the expanded 1024-QAM modulation scheme with three features. First, we insert only $524 \mathrm{Kbps}$ pilot symbols to overcome DVB-C2 ensemble echo channel. Second, we adopt transport stream layer FEC (TS-FEC) to recover the burst noise error. Third, we reuse almost all components of the system described in OpenCable (ITU-T J.93 Annex B) to resolve the hardware implementation issue.

This paper is organized as follows. In section 2. a brief description of overall system is given first, followed by the functional description of each module. Section 3. describes performance of proposed system with computer simulation results, then laboratory test results of prototype $\mathrm{H} / \mathrm{W}$ are presented in section 4., finally some conclusion presented in section 5 .

\section{SYSTEM DESCRIPTION}

The problem not resolved in previous researches is to provide additional features for ultra-high density television (UHDTV) with ease of hardware implementation. The additive features for UHDTV are higher data rate, and higher coding gain. In previous researches, to provide the additive features, many components of systems have been changed[3][4]. In our research, almost all components of the system described in OpenCable (ITU-T J.83 annex B) are reused. The reuse philosophy in our research resolves the hardware implementation issue. For an adaptive system, the data rate changes adaptively to the channel state. In other words, an adaptive system operates with low data rate when the channel is in the bad state. In converse, this system operates with high data rate when the channel is in the good state. In previous researches, the system should have more than two different modems, one for the good channel state to provide additive features for UHDTV, and the others for the bad channel states. In our research, almost all of the modem components are reused for the various data rates. In detail, we reuse the $(128,122)$ encoder, convolutional interleaver, randomizer, and convolutional encoder of TCM encoder. TCM encoder is slightly modified to use 1024-QAM signal 
constellation. The new 1024-QAM signal constellation in our research succeeds the features of the previous 256-QAM

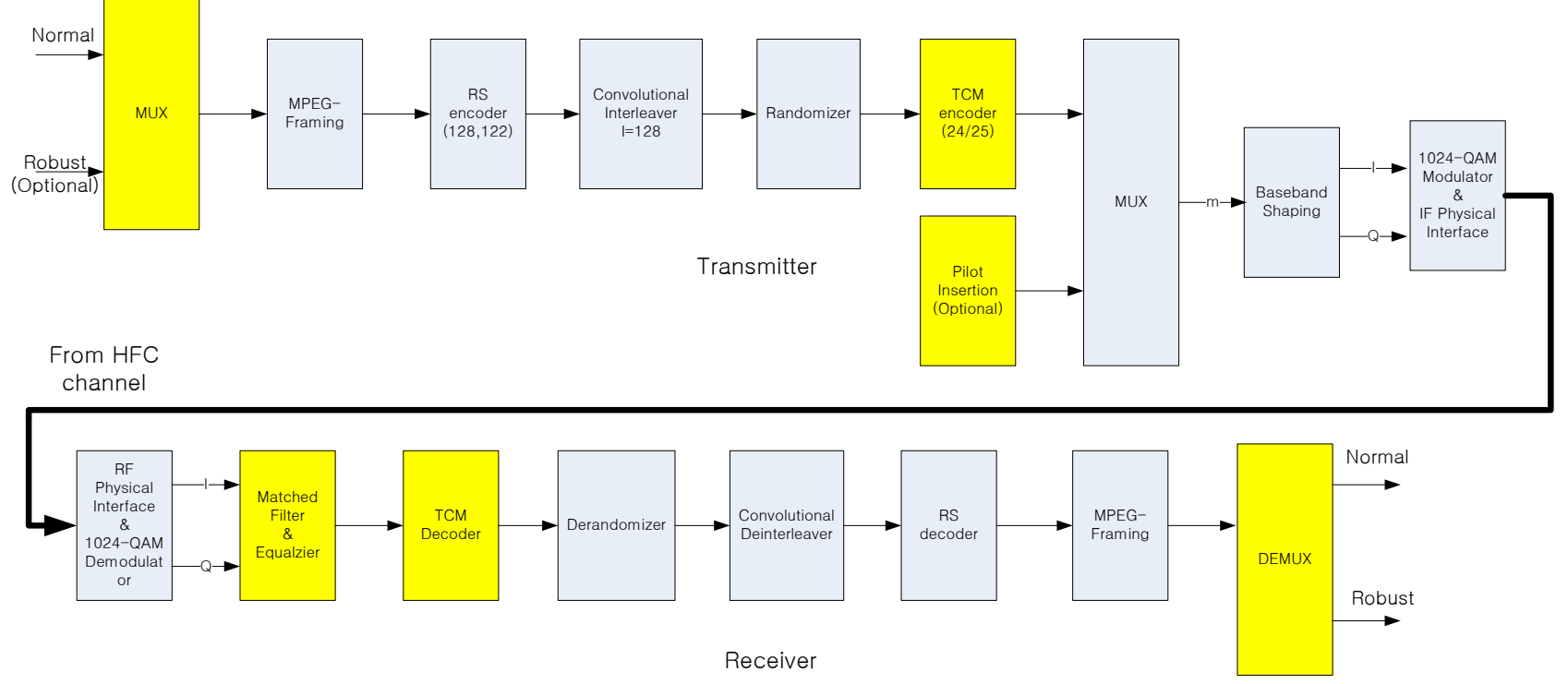

Figure 1. Block diagram of Expanded OpenCable(J.83 Annex B) 1024-QAM transmitter and receiver system

signal constellation such as 90 degree rotationally invariant property, and bit labeling, so 1024-QAM modulator and demodulator can reuse those of 256-QAM. Functional block diagram for the proposed downlink system is shown in Figure 1. The main features of proposed downlink schemes are expanded OpenCable(J.83 Annex B) modem design, single carrier modulation applied 1024-QAM in $6 \mathrm{MHz}$ bandwidth, RS outer coder, convolutional interleaver, TCM inner coder for physical layer FEC, TS-FEC and pilot sequence insertion for against burst noise, fast symbol time/frequency carrier recovery and channel estimation. Except TS-FEC multiplexer/demultiplexer, pilot sequence insertion and slightly modified TCM encoder, almost components of OpenCable(J.83 Annex B)64/256-QAM modem could be reused for the proposed 1024-QAM implementation[1].

\section{A. TS-FEC}

TS-FEC provides higher coding gain, layer transparency, and backward compatibility. Higher coding gain is achieved by a forward error correction (FEC) code such as $\mathrm{RS}$ code. Layer transparency and backward compatibility are achieved by MPEG-2 TS packetizing. An MPEG-2 TS packet is composed of 4 bytes header and 184 bytes payload. Header bytes are composed of 1 byte sync pattern and 3 bytes control information. 3 byte control information represents $2^{24}$ values. Some of the values are defined for the controlling of packet in the MPEG-2 TS format but some of the values are not defined yet. Generally, the packet including the undefined control information is discarded at the receiver. TS-FEC uses one of the undefined control information, say unique PID, to identify the parity packets provided to protect payload information packets from errors. These parity packets are meaningless with normal receiver which has no information about the unique PID, but the enhanced receiver which knows the

unique PID uses the parity packets to correct the errors in payload information packets. The robust stream protected by using TS-FEC with $\operatorname{RS}(255,239)$ satisfies TOV(Threshold Of Visibility, BER of 3.6e-6) at $32.2 \mathrm{~dB}$ of CNR. In this case, the overall code rate is $(48 / 50)(239 / 255)$ $=8.9976$

\section{B. TS-FEC MUX and MPEG-2 TS framing}

TS-FEC MUX provides a multiplexing robust stream with TS-FEC and normal stream. MPEG-2 TS framing is exactly same as J.83 Annex B MPEG framing.

\section{Reed-Solomon Encoder}

A systematic encoder is utilized to implement at $\mathrm{t}=3,(128$, 122) extended Reed-Solomon code over GF(128). The primitive polynomial used to form the field over GF (128) is :

$$
p(x)=x^{7}+x^{3}+1
$$

where: $p(\alpha)=0$

the generator polynomial used by the encoder is :

$g(x)=(x+\alpha)\left(x+\alpha^{2}\right)\left(x+\alpha^{3}\right)\left(x+\alpha^{4}\right)\left(x+\alpha^{5}\right)$

This block is exactly same as J.83 Annex B RS encoder. 


\section{Convolutional Interleaver}

Interleaving is included in the modem between the RS block coding and randomizer to enable the error correction of burst noise induced errors. Figure 2. shows a block diagram of convolutional interleaver and deinterleaver. This block is exactly same as J.83 Annex B convolutional interlaver and deinterleaver

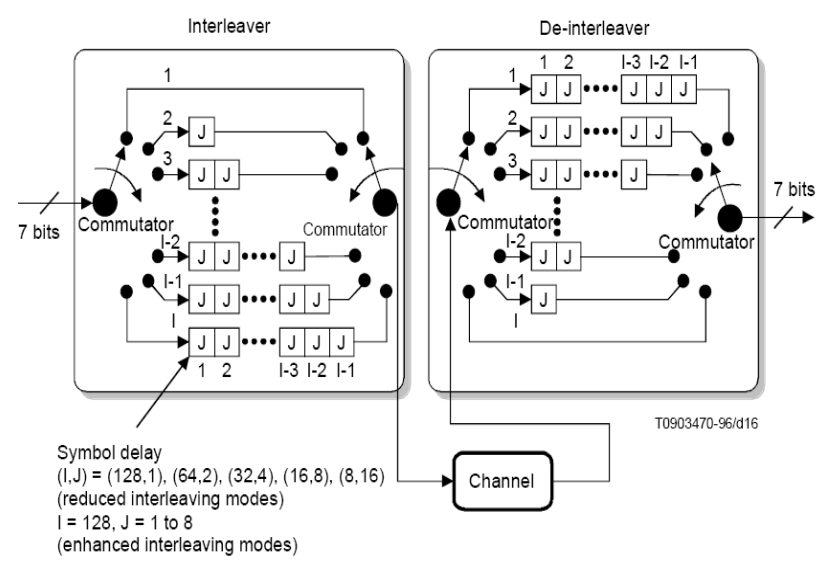

Figure 2. Convolutional interleaver-deinterleaver

\section{E. Randomizer}

The randomizer provides for even distribution of the symbols in the constellation, which enables the demodulator to maintain proper lock. The randomizer adds a Pseudorandom Noise (PN) sequence of 7-bit symbols over GF(128) to the symbols. The randomizer users a liner feedback shift register specified by a GF(128) polynomial defined as follows:

$$
f(x)=x^{3}+x+\alpha^{3}
$$

where:

$$
\alpha^{7}+\alpha^{3}+1=0
$$

This block is exactly same as J.83 Annex B randomizer.

\section{F. TCM Encoder}

For 1024-QAM, an analogous trellis coding is employed using the same Binary Convolutional Coder (BCC) as 64QAM and 256-QAM, with the same rate $1 / 2$ generator and the same 4/5 puncture matrix as J.83 Annex B trellis encoder. Of the 48 input bits that form a trellis group, each of two groups of 4 bits of the differentially pre-code bit streams in a trellis group are separately encoded by a BCC. Each BCC produces 5 coded bits. The remaining bits are sent to the QAM mapper uncoded. This produces a total output of 50 bits per trellis group. Thus, the overall code rate for 1024-QAM trellis coded modulation is $24 / 25$. Figure 3 shows the 1024-QAM trellis coded modulator block diagram.

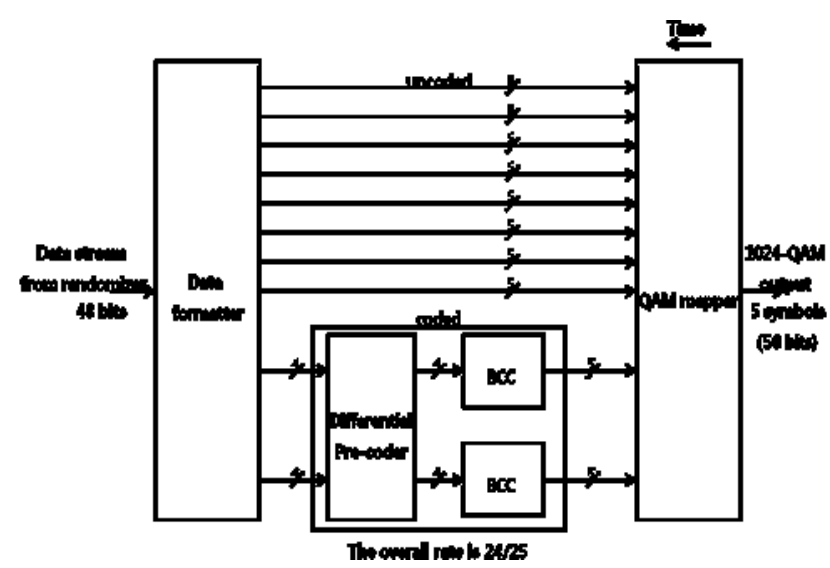

Figure 3. 1024-QAM trellis coded modulator block diagram

The differential pre-coder shown in Figure 3 performs the 90 degree rotationally invariant trellis coding. This block is slightly modified for 1024-QAM which is compared to J. 83 Annex B. The BCC is a punctured rate $1 / 2$ binary convolutional encoder. The convolutional encoder is a 16 -sate non-systematic rate $1 / 2$ encoder with the generator: $\mathrm{G} 1=010$ $101, \mathrm{G} 2=011111(25,37$ octal $)$. The output of the encoder is selected according to a puncture matrix: $[\mathrm{P} 1, \mathrm{P} 2]=[0001$; 1111] ("0" denotes NO transmission, " 1 " denotes transmission), which produces a single serial bit stream. The puncture matrix essentially converts the rate $1 / 2$ encoder to rate $4 / 5$, since only 5 of the 8 encoded bits are retained after puncturing. This block is slightly modified for 1024-QAM which is compared to J. 83 Annex B.

\section{G. Pilot Insertion}

This unit shall perform a pilot sequence providing for fast symbol time/frequency recovery and channel estimation. 1\% (524Kbps for 1024-QAM) pilot symbols per total payload will be inserted

\section{H. Baseband Shaping}

This unit shall perform mapping from $\mathrm{m}$-tuples to I and Q signals and a square-root raised cosine filtering of the I and Q signals prior to QAM modulation

\section{QAM modulation and physical interface}

This unit performs QAM modulation. It is followed by interfacing the QAM modulated signal to RF cable channel

Receiver system shall perform the inverse signal processing, as described for modulation process above, in order to recover the baseband signal 


\section{III.PERFORMANCE OF PROPOSED SYSTEM}

The proposed system is simulated under AWGN and multipath environment to measure the TOV. Table 1 shows the performance comparison of different constellation under AWGN channel. The testing is to determine any changes in the robustness to random noise impairment. The noise level is increased until TOV is reached and Es/No value is recorded in the table 1 .

Table 1. Performance of 1024-QAM under AWGN

\begin{tabular}{|c|c||c||}
\hline $\begin{array}{c}\text { Modulation } \\
\text { Type }\end{array}$ & $\begin{array}{c}\text { Data Rate } \\
(6 \mathrm{MHz} \mathrm{BW})\end{array}$ & Es/No@TOV(dB) \\
\hline \hline 1024-QAM & $48.54 \mathrm{Mbps}$ & $32.7 \mathrm{~dB}$ \\
\hline
\end{tabular}

Figure 4 shows the BER performances of TCM outputs. TCM output bits are fed into the RS decoder to correct errors after Viterbi decoding. RS $(128,122)$ decoder output streams achieve TOV (3.6e-6) when the BER performance of TCM outputs is less than 4.6e-4. TCM applied to 64-QAM and TCM applied to 256-QAM are exactly same as described in J.83 Annex B. The block diagram of TCM applied to 1024QAM is depicted in Figure 3.[3][4]

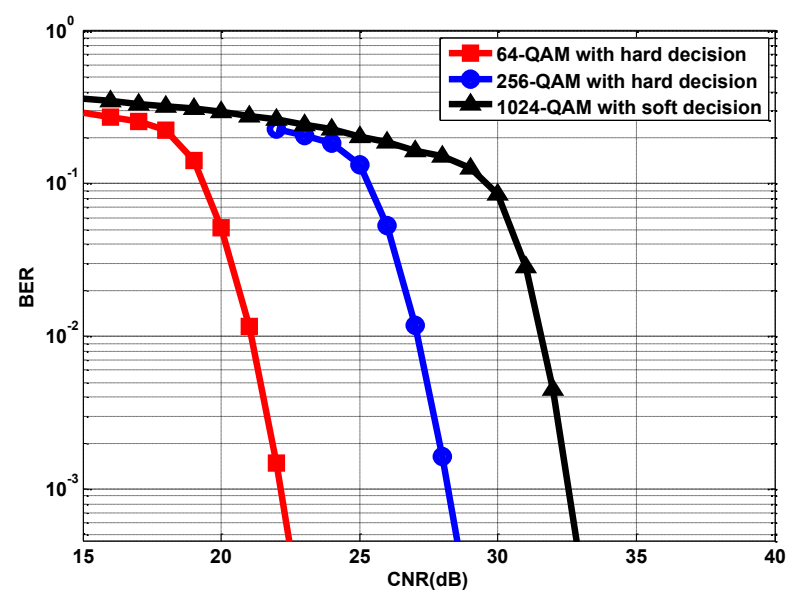

Figure 4. BER curves of TCM outputs

In the OpenCable(J.83 AnnexB) standard, blind DFE (Decision Feedback Equalizer) is usually used to compensate a multipath channel distortion at receiver side. But in the severe multipath channel condition, high order QAM performance limitation by distortion of input symbol constellation and increased probability errors, leading to error propagation in DFE. By inserting pilot symbols which account for only $1 \%$ (524kbps PN sequence for $1024-$ QAM) to total amount of transmission data and number of taps for feed-forward are 40, feed-back are 40, the proposed system receiver can be significantly improved ghost handling performance. Table 2 shows the performance comparison of different constellation under strong static multipath channel. The purpose of this testing is to determine any changes in robustness to static single/ensemble multipath channel impairment with random noise injection. and Table 3 shows channel profile of DVB-C2 \#1.2, \#2.2 ensemble echo. It can be observed that proposed system is strong enough to overcome severe echo channel on HFC network. Figure 5 shows spectrum of transmitted signal before and after multipath distortion. The input $\mathrm{SNR}$ is $35 \mathrm{~dB}$, modulation is $1024-\mathrm{QAM}$ mode, $-3 \mathrm{~dB}$ $5 \mu$ s post single echo case[5][6].

Table 2. Performance of 1024-QAM under Multipath fading channel

\begin{tabular}{||c|c|c|c||}
\hline $\begin{array}{c}\text { Modulation } \\
\text { Type }\end{array}$ & Channel & $\begin{array}{c}\text { SNR } \\
(\mathrm{dB})\end{array}$ & Results \\
\hline \hline 1024-QAM & $\begin{array}{c}-3 \mathrm{~dB}, 5 \mu \mathrm{S} \\
\text { single echo }\end{array}$ & 35 & Pass \\
\hline 1024-QAM & $\begin{array}{c}\text { DVB-C2 \#1.2 } \\
\text { ensemble }\end{array}$ & 42 & Pass \\
\hline 1024-QAM & $\begin{array}{c}\text { DVB-C2 \#2.2 } \\
\text { ensemble }\end{array}$ & 45 & Pass \\
\hline
\end{tabular}

Table 3. Channel profile of DVB-C2 \#1.2, \#2.2 ensemble echo

\begin{tabular}{|c|c|c|c|c|c|c|c|c|}
\hline \multirow[t]{2}{*}{ Path } & \multicolumn{2}{|c|}{$\begin{array}{c}\text { Delay } \\
(\mu \mathrm{s})\end{array}$} & \multicolumn{2}{|c|}{$\begin{array}{c}\text { Echo } \\
\text { Amplitude } \\
(\mathrm{dB}) \\
\end{array}$} & \multicolumn{2}{|c|}{$\begin{array}{c}\text { Phase } \\
\text { (П) }\end{array}$} & \multicolumn{2}{|c|}{$\begin{array}{l}\text { SNR } \\
(\mathrm{dB})\end{array}$} \\
\hline & $\# 1.2$ & $\# 2.2$ & $\# 1.2$ & \#2.2 & $\# 1.2$ & $\# 2.2$ & $\# 1.2$ & $\# 2.2$ \\
\hline 1 & \multicolumn{2}{|c|}{0} & \multicolumn{2}{|c|}{0} & \multicolumn{2}{|c|}{0} & \multirow{7}{*}{42} & \multirow{7}{*}{45} \\
\hline 2 & \multicolumn{2}{|c|}{0.038} & -14 & -11 & \multicolumn{2}{|c|}{0.95} & & \\
\hline 3 & \multicolumn{2}{|c|}{0.181} & -17 & -14 & \multicolumn{2}{|c|}{1.67} & & \\
\hline 4 & \multicolumn{2}{|c|}{0.427} & -23 & -17 & \multicolumn{2}{|c|}{0.26} & & \\
\hline 5 & \multicolumn{2}{|c|}{0.809} & -32 & -23 & \multicolumn{2}{|c|}{1.20} & & \\
\hline 6 & \multicolumn{2}{|c|}{1.633} & -40 & -32 & \multicolumn{2}{|c|}{1.12} & & \\
\hline 7 & \multicolumn{2}{|c|}{3.708} & -51 & -40 & \multicolumn{2}{|c|}{0.81} & & \\
\hline
\end{tabular}

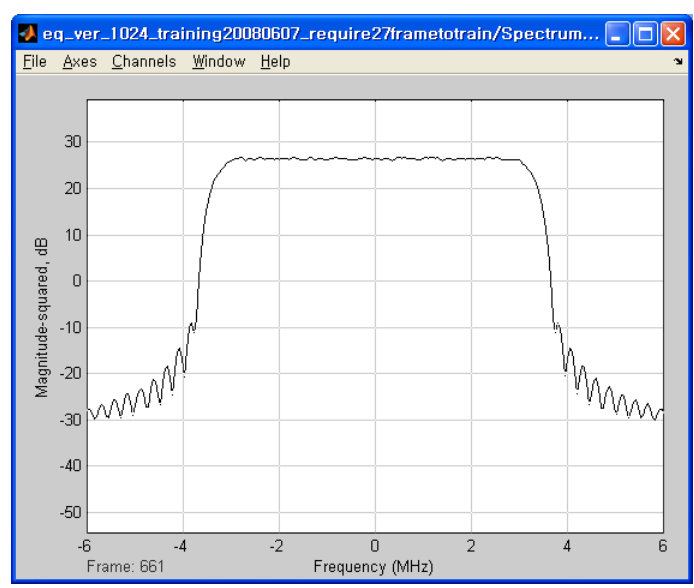

(a) Spectrum of Transmitted Signal before HFC Multipath Channel 


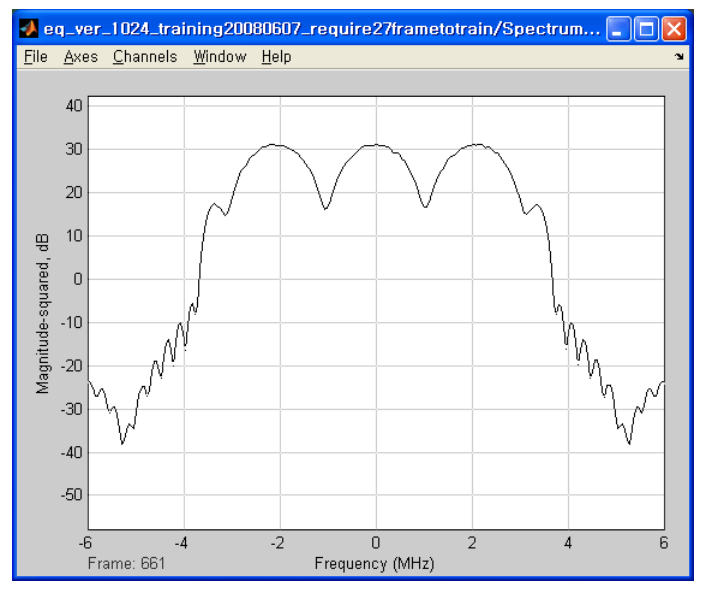

(b) Spectrum of transmitted signal after HFC multipath channel

Figure 5. Power spectrum of transmitted signal with fading distortion

Figure 6. shows transmitted, before equalized received signal, after equalized received constellations with input SNR $35 \mathrm{~dB},-3 \mathrm{~dB} 5 \mu \mathrm{s}$ post single echo distortion for 1024-QAM mode. It can be observed that equalization algorithm with pilot symbol is working successfully on HFC server channel environment [7][8][9][10].

Figure 7 shows a comparison of a equalizer convergence speed between pilot based equalization to blind equalization algorithm. when the input SNR is $35 \mathrm{~dB},-3 \mathrm{~dB} 5 \mu$ s post single echo case. It can be observed that pilot based DFE successfully compensates distortion by reducing error propagation and significantly increases convergence speed, compared to blind DFE[11][12].

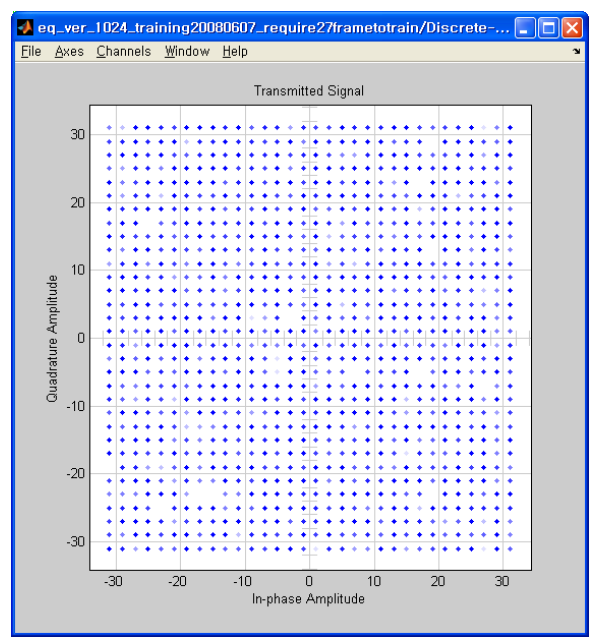

(a) 1024-QAM transmitted singnal constellation without multipath channel distortion

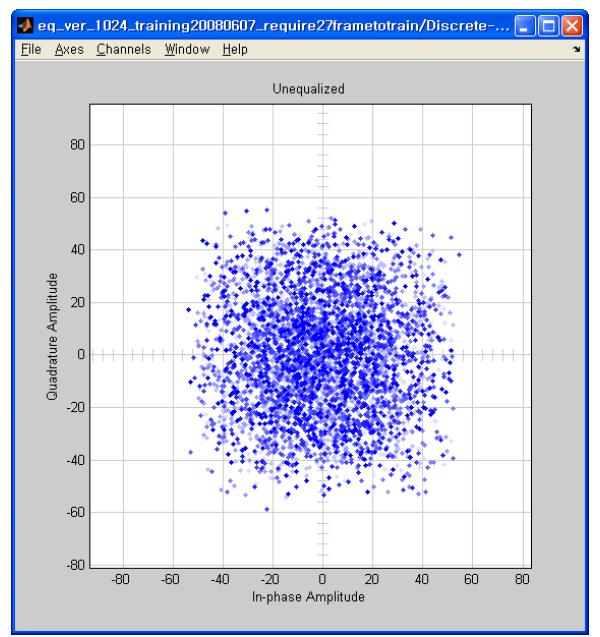

(b) 1024-QAM received signal constellation before equalization

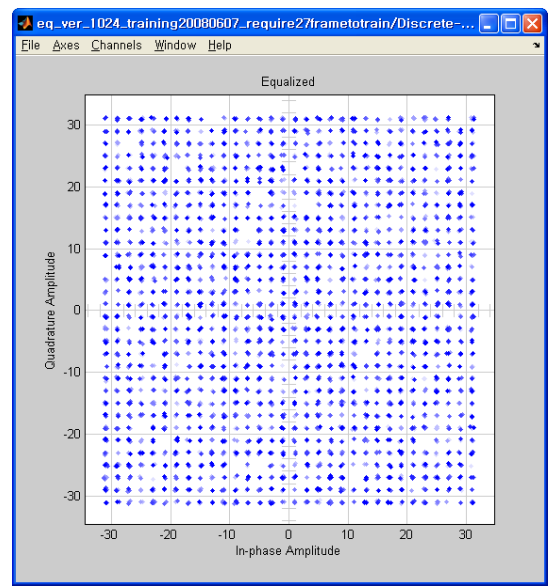

(c) 1024-QAM received signal constellation after equalization

Figure 6. Performance of 1024-QAM pilot based equalization under strong static echo channel

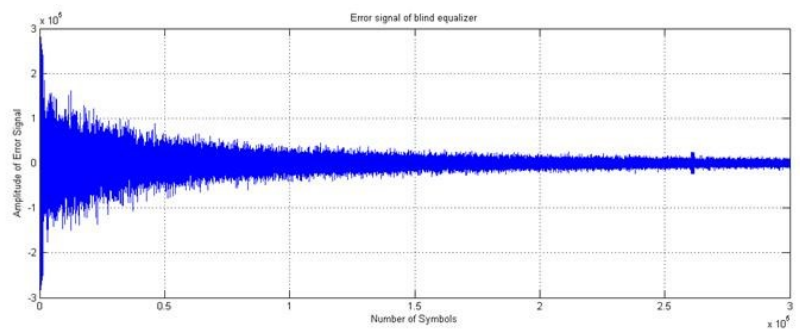

(a) Error signal of blind equalizer 


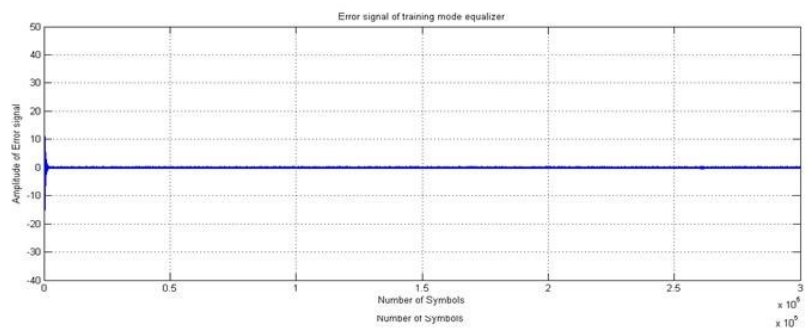

(b) Error signal of pilot based equalizer

Figure 7. Equalizer convergence speed comparison between blind and pilot based algorithm

\section{IV.1024-QAM LABORATORY TEST}

The 1024-QAM test bed set-up for $\mathrm{H} / \mathrm{W}$ performance evaluation is shown in Figure 8.

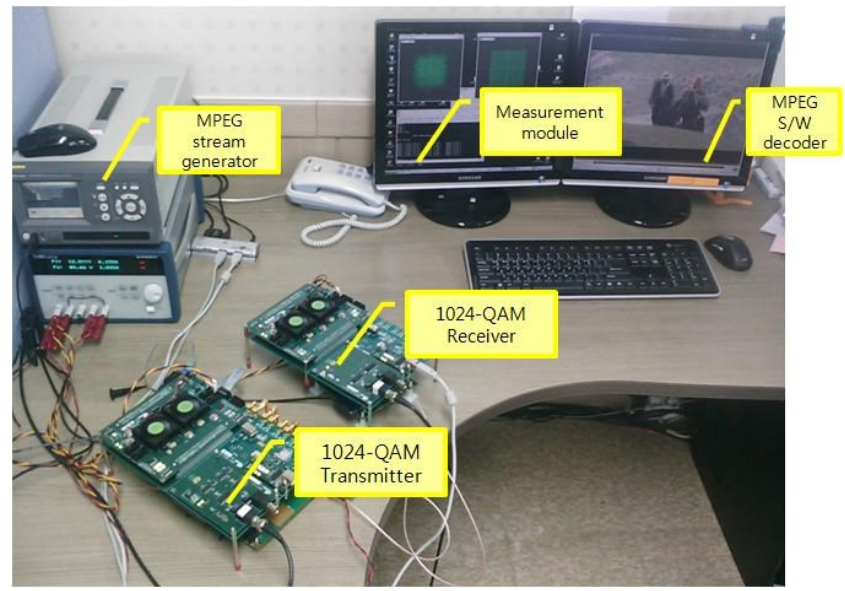

Figure 8. 1024-QAM Test Bed

1024-QAM test bed consists of MPEG stream generator, 1024-QAM transmitter and receiver, AWGN noise generator, fading channel simulator, 1024-QAM measurement equipment for transmission and reception signal quality measurement, MPEG-2 S/W Demux/Decoder. A prototype 1024-QAM transmitter and receiver were developed to verify computer simulation results which described in section 3 .

Figure 9, Figure 10 show the 1024-QAM reception signal spectrum, symbol constellations before equalization and after equalization under DVB-C2 \#1.2, DVB-C2 \#2.2 fading channel model when the input SNR is $40 \mathrm{~dB}$. This testing results indicate that 1024-QAM transmitter and recever is working successfully at DVB-C2 \#1.2, DVB-C2 \#2.2 fading channel codition.

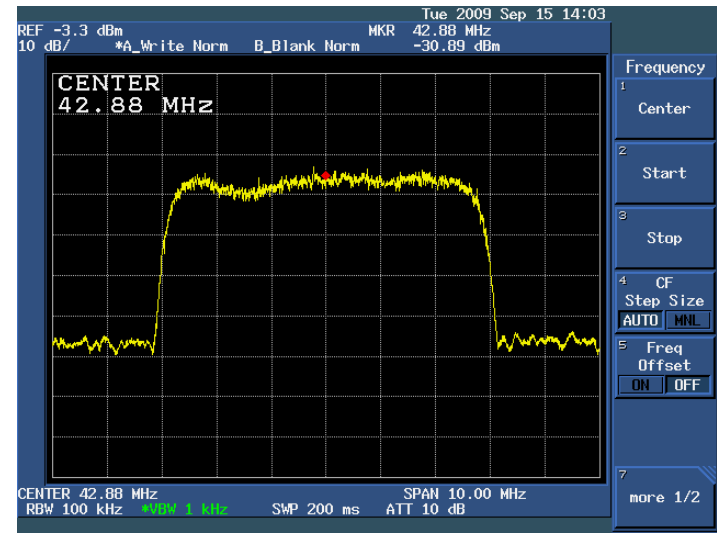

(a) Spectrum of 1024-QAM received signal under DVB-C2\#1.2 fading channel

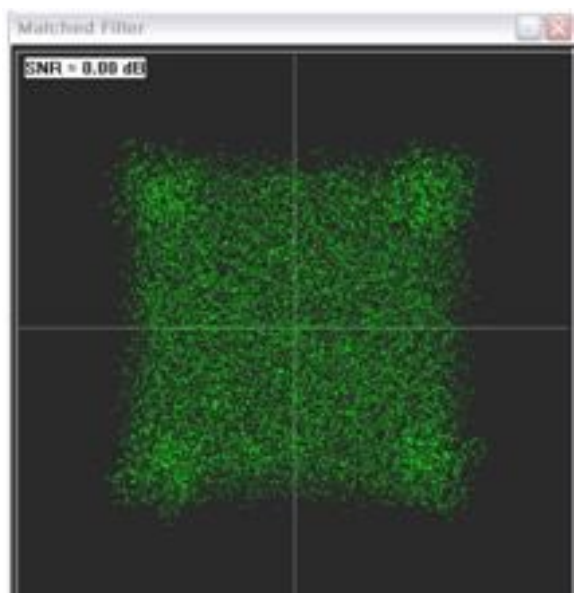

(b) Symbol constealltion of 1024-QAM received signal before equalization(DVB-C\#1.2 SNR 40dB)

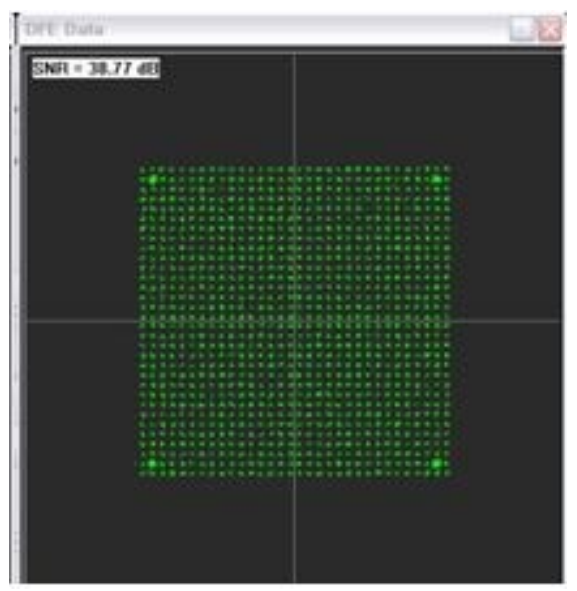

(c) Symbol constellation of 1024-QAM received signal after equalization(DVB-C2\#2.2 SNR 40dB)

Figure 9. 1024-QAM signal mesuring under DVB-C2\#.12 fading channel 


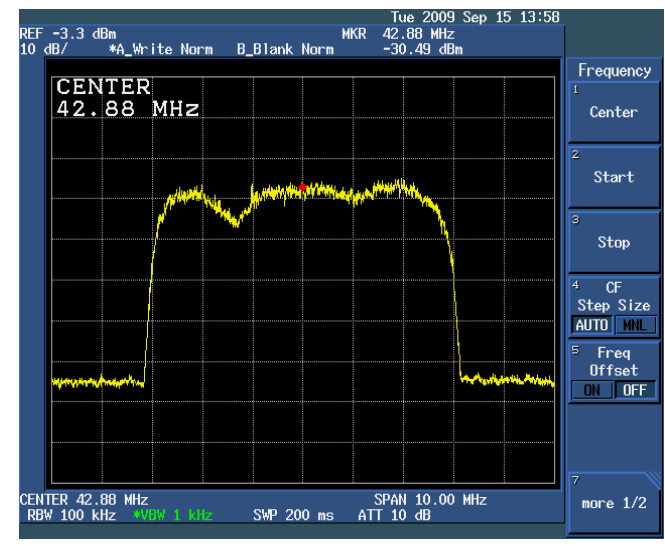

(a) Spectrum of 1024-QAM transmitted singnal under DVB-C2\#2.2 fading channel

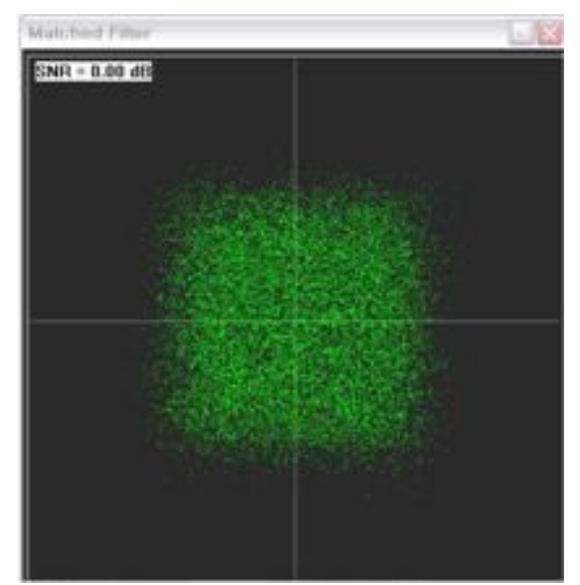

(b) Symbol constealltion of 1024-QAM received signal before equalization(DVB-C\#2.2 SNR 40dB)

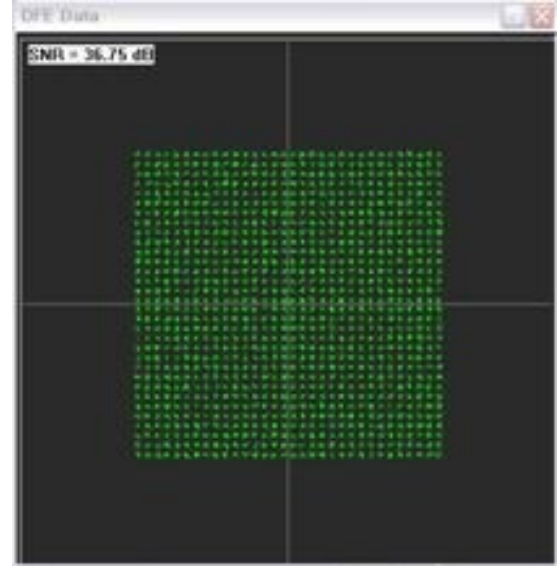

(c) Symbol constellation of 1024-QAM received signal after equalization(DVB-C2\#2.2 SNR 40dB)

Figure 10. 1024-QAM signal mesuring under DVB-C2\#.12 fading channel

\section{Conclusions}

This paper describes a cost effective expanded OpenCable (J.83 Annex B) 1024-QAM transmission system design for high quality band intensive services. The proposed system requires about $32.7 \mathrm{~dB}(\mathrm{Es} / \mathrm{No})$ for 1024 QAM signal reception to TOV under AWGN and provides a great ghost and burst noise handling performance with an insertion of small amount of pilot symbols and TS-FEC. We proposed single carrier transmission system based on TS-FEC and pilot based channel equalization for $4 \mathrm{~K}$ UHDTV services within $6 \mathrm{MHz}$ one channel and $8 \mathrm{~K}-$ UHDTV could be supported by multiple broadcasting channel bonding on HFC network.

\section{ACKNOWLEDGMENT}

"The work was supported by the IT R\&D program(2008-F011, Development of Next Generation DTV Core Technology) of KEIT\&KCC\&MKE, Korea. \& [Development of DVB-T2 Fixed/Mobile TV Hybrid High Quality 3DTV Multiplexer and Receiver System, N011500052]

\section{REFERENCES}

[1] ITU-T Recommendation J.83, "Digital Multi-program Systems for Television Sound and Data Services for Cable Distribution," April 1997.

[2] DVB-CM, "Advanced Coding and Modulation Schemes for CATV systems(DVB-C2)," December 122007.

[3] Chang Yeon Cho, Jun Heo, Joon Tae Kim, "An Extension of J.83 Annex B Transmission Systems for Ultra-High Definition (UD) TV Broadcasting", IEEE Transaction on Consumer Electronics, Vol. 55, No.1, pp. 63-68, February. 2009.

[4] Kamilo Feher, "1024-QAM and 256-QAM Coded Modems for Microwave and Cable system Applications", IEEE Journal on Selected on Areas in Communications, April 1997.

[5] Shafayat Abrar and Roy A. AxfordJr, "Sliced Multi-modulus Blind Equalization Algorithm ”, ETRI Journal, Vol.27, No. 3. pp. 257-266, June 2005.

[6] ReDesign, "HFC Channel Model" DVB-TM C2 196 December 2008

[7] Takuya KURAKAKE, Naoyoshi NAKAMURA and Kimiyuki OYAMADA, "A Blind 1024-QAM Demodulator for Cable Television, "Int Zurich Seminar on Communications, Vol.1, pp. 136138, February 2004

[8] Franco Mazzenga, "Channel Estimation and Equalization for M-QAM Transmission with a hidden pilot sequence " IEEE Transaction on Broadcasting, Vol.46, No.2. pp. 170-176, June 2000.

[9] Ki Seol Kim, Kwangmin Hyun, Chang Wahn Yu, Youn Ok Park, Dongweon Yoon and Sang Kyu Park, "General Log-Likelihood Ratio Expression and Its Implementation Algorithm for Gray-Coded QAM Signals" ETRI Journal, Vol.28, No.3. pp. 291-300, June 2006.

[10] SeungWon Kang, KyunHi Chang, "A Novel Channel Estimation Scheme for OFDM/OQAM-IOTA System" ETRI Journal, Vol.29, No.4. pp. 430-436, August 2007.

[11] Dongweon Yoon, Dae-Ig Chang, Nae-Soo Kim, and Hoon-Shik Woo, "Linear Diversity Analysis for M-ary Square Quadrature Amplitude Modulation over Nakagami Fading Channel" ETRI Journal, Vol.4, No.4. pp. 231-237, August 2003.

[12] Chang-Joo Kim, Young-Su Kim, Goo-Young Jeong, Jae-Kyung Mun, and Hyuck-Jae Lee, "SER Analysis of QAM with Space Diversity in Rayleigh Fading Channels" ETRI Journal, Vol.17, No.4. pp. 25-35, January 1996. 


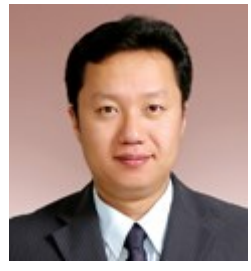

Sung-Hoon Kim received B.S., M.E., and Ph.D. degrees in electronics engineering from Kookmin University, Seoul, KOREA in 1994, 1996 and 2008 , respectively. since 2000 , he has been a Principal Researcher in Electronics and Telecommunications Research Institute (ETRI), Korea. His research interests are digital signal processing in field of Terrestrial \& Mobile DTV, 3DTV and UHDTV broadcasting system

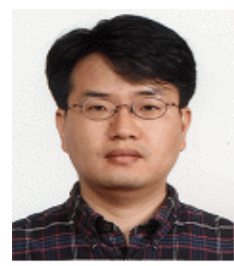

Jin Soo Choi received the B.E., M.E., and Ph.D. degrees in electronic engineering from Kyungpook National University, Korea, in 1990, 1992, and 1996, respectively. Since 1996, he has been a principal member of engineering staff in Electronics and Telecommunications Research Institute (ETRI), Korea. He has been involved in developing MPEG4 codec system, data broadcasting system, and 3D/UHDTV broadcasting system. His research interests include visual signal processing and interactive services in the field of the digital broadcasting technology.

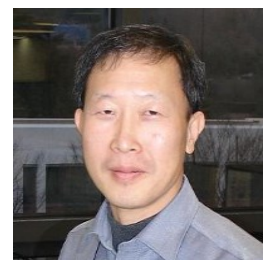

Jinwoong Kim received the BS and MS degrees in electronics engineering both from Seoul National University, Seoul, Korea, in 1981 and 1983. He received the $\mathrm{PhD}$ degree in electrical engineering from Texas A\&M University, Texas, USA, in 1993. He has been with ETRI since 1983, and is a principal researcher and managing director in Broadcasting and Telecommunications Media Research Laboratory. His area of interest includes digital broadcasting system, digital video processing, 3DTV, UHDTV and digital holography.

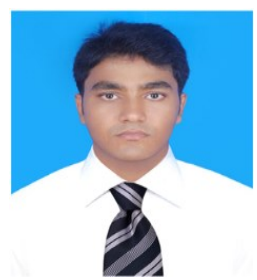

Md. Sazzad Hossen received his B.Sc. in Electrical and Electronic Engineering from Khulna University of Engineering and Technology, Bangladesh in 2012. In 2013, he joined the Multimedia Communication and Signal Processing (MCSP) lab as a research student in the department of Electronics Engineering at Kookmin University, South Korea. His research interests include indoor positioning, digital video broadcasting, DVB-T2, 3DTV broadcasting system and OFDM system.

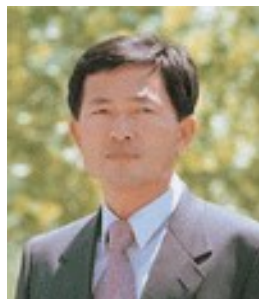

Ki-Doo Kim received the B.S. degree in electronics engineering from Sogang University, Seoul,Korea, in 1980, and the M.S. and Ph.D. degrees from Pennsylvania State University, University Park, in 1988 and 1990, respectively, both in electrical engineering. From 1980 to 1985, he was a Research Engineer at the Agency for Defense Development in Korea. In March 1991 he joined the Department of Electronics Engineering, Kookmin University, Seoul, Korea, and is currently a Professor. He worked as a Visiting Scholar in the Department of Electrical and Computer Engineering, University of California San Diego, from February 1997 to February 1998. His current research interests are digital broadcasting systems and mobile communications. 